FRIEDRICH HILD / WIEN

\title{
LYKIEN IN DEN NOTITIAE EPISCOPATUUM
}

Die Notitiae episcopatuum sind die wichtigste Quelle für die kirchliche Geographie des Byzantinischen Reiches ${ }^{1}$. Es sind Verzeichnissse der Metropolien, der autokephalen Erzbistümer und der zu den Metropolen gehörenden Suffraganbistümer, die für Zwecke des Protokolls geschaffen wurden. Sie spiegeln immer wieder Änderungen im Gesamtbestand des Episkopats durch den Bedeutungsverlust oder -gewinn einzelner Bistümer im Verlauf der politischen Geschichte wider. Gelegentlich stellen die Notitiae jedoch nur noch Ansprüche und keine Wirklichkeit dar. Sie müssen daher durch die Geschichte ihrer Entstehungszeit, unter anderem durch die Konzilsakten, überprüft werden ${ }^{2}$. In manchen Fällen werden die Notitiae auch zu einer wichtigen Quelle für die politische Geschichte der Provinz, insbesondere für die Siedlungsgeschichte, wie im Falle der Kirchenprovinz Lykia.

Auffällig ist bei den Notitiae von Lykien zunächst die hohe Zahl von 37 Bistümern in einer relativ kleinen Provinz mit nur ca. $12.500 \mathrm{~km}^{2}$ Grundfläche. Im benachbarten Pamphylien (Provinzen Pamphylia I und II) kommen auf $16.500 \mathrm{~km}^{2} 35$ Bistümer (in Lykien hat demnach im Schnitt ein Bistum $330 \mathrm{~km}^{2}$, in Pamphylien $470 \mathrm{~km}^{2}$ ). Im krassen Gegensatz stehen dazu etwa die Provinzen Paphlagonia und Honorias (TIB 9) mit $46.000 \mathrm{~km}^{2}$ und nur 10 Bistümern. Hier kommen auf ein Bistum ca. $4.600 \mathrm{~km}^{2}$.

Besonders bemerkenswert ist dann die Rangordnung der Bistümer. Während in den Notitiae der meisten Provinzen die schon in der Antike bedeutenden Städte auch hohen Rang aufweisen, ist das in Lykien anders. Als Protothronos der Metropole Myra erscheint ein in antiken Quellen nicht bezeugter Ort: Mastaura.

${ }^{1}$ Die Notitiae des Patriarchats Konstantinopel sind ediert von J. DArrouzès, Notitiae episcopatuum Ecclesiae Constantinopolitanae (Géographie ecclésiastique de l'empire byzantin 1). Paris 1981.

${ }^{2}$ H.-G. BEcK, Kirche und theologische Literatur im byzantinischen Reich (HdA XII 2/1). München 1959, 148; vgl. auch A. Kazhdan, Notitiae episcopatuum. ODB III (1991) 1496. - Eine Übersicht über die Rangordnung der lykischen Bistümer in den Notitiae und Konzilsakten bietet bereits J. Darrovzès, Sur les variations numériques des évêchés byzantins. REB 44 (1986) $18 f$. 
Im folgenden soll versucht werden, anhand der Lemmata im Band 8 der Tabula Imperii Byzantini ${ }^{3}$ und der zwei Tabellen (Notitiae episcopatuum und Konzilsakten ${ }^{4}$ ) die Rangordnung in den Notitiae Lyciae vorzustellen und, wenn möglich, zu erklären.

Schon die Stellung Myras als Metropole der Provinz ist erstaunlich, war doch Patara die Hauptstadt der 43 n. Chr. eingerichteten römischen Provinz Lycia, die 73/74 n. Chr. mit Pamphylia zu einer Doppelprovinz vereinigt wurde. Am ersten ökumenischen Konzil von Nikaia nahm 325 n. Chr. Bischof Eudemos von Patara als einziger lykischer Bischof teil, Patara könnte demnach damals noch die Hauptstadt der seit der diokletianischkonstantinischen Provinzialreform wieder selbständigen Provinz Lykia gewesen sein. Myra war allerdings eine der sechs in der römischen Kaiserzeit inschriftlich bezeugten Metropolen Lykiens (außer Myra: Patara, Telmessos, Xanthos, Tlos und Limyra). Bischof Tatianos von Myra steht 381 in den Konzilsakten des ersten ökumenischen Konzils von Konstantinopel an erster Stelle der lykischen Bischöfe (vor Choma und Patara); unter Theodosios II. (408-450) wurde Myra Hauptstadt der Provinz Lykia. Die Spitzenstellung Myras ist daher historisch begründet.

Man würde nun aber an zweiter Stelle als Protothronos des Provinzialepiskopats Patara erwarten. An seiner Stelle führt jedoch Mastaura in allen Notitiae bis in das 12. Jahrhundert die Liste der lykischen Bischöfe an. Patara hingegen erscheint vom 7 . bis in das 10. Jahrhundert an 31./ 32. Stelle. Der Niedergang Pataras zeichnet sich schon im 6. Jahrhundert ab: 537 verbannte Belisar Papst Silverius nach Patara; da sich der Bischof von Patara für ihn einsetzte, kehrte er auf Weisung Kaiser Iustinians I. nach Italien zurück, wo er noch 537 - neuerlich verbannt - auf der Insel Ponza (Palmaria) im Golf von Gaeta starb ${ }^{5}$. Nach einer zwischen 750 und 850 geschriebenen Vita des in Patara geborenen H. Nikolaos von Myra war

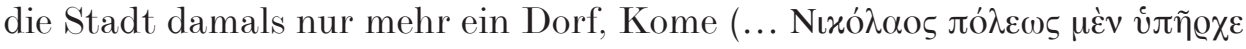

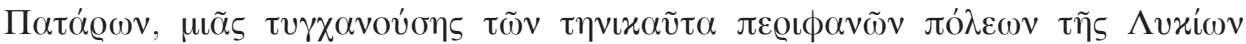

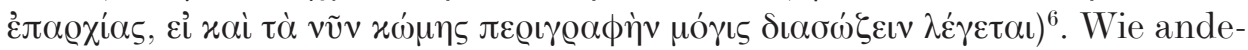

${ }^{3}$ H. Hellenkemper - F. Hild, Lykien und Pamphylien. TIB 8. Wien 2004.

${ }^{4}$ Belege in TIB 8 bei den einzelnen Lemmata; zu den Akten des Konzils von $787 \mathrm{vgl}$. nun auch E. Lamberz, Die Bischofslisten des VII. ökumenischen Konzils (Nicaenum II). Abhandlungen, phil.-hist. Kl., Bayer. Akad. Wiss., N. F. 124. München 2004.

${ }^{5}$ Liberati Breviarium, ACO II 5, 137; G. Schwaiger, Silverius, Papst. LThK 9 (2000) 586 .

${ }^{6}$ G. Anrich, Hagios Nikolaos. Der heilige Nikolaos in der griechischen Kirche, I. Die Texte, II. Prolegomena, Untersuchungen, Indices. Leipzig-Berlin 1913-1917, I, 114, II $262 \mathrm{f}$. 
re lykische Hafenorte, so vor allem Phaselis und Olympos, hatte Patara kein landwirtschaftlich nutzbares Hinterland und lebte vorwiegend von Seefahrt und Seehandel. Bis zur arabischen Eroberung Ägyptens 642 n. Chr. beruhte die Lebensmittelversorgung Konstantinopels wesentlich auf den jährlichen Getreidelieferungen aus Ägypten, für welche die Granarien in Andriake und Patara wichtige Zwischenstationen waren.

Damals, im 7. Jahrhundert, als die erste Notitia Lykiens entstand, verlor Patara auch diese Funktion. In den Akten des zweiten ökumenischen Konzils von Nikaia (787) erscheint Patara noch an zweiter Stelle nach Myra, in den Akten von 879 nur mehr an achter. Patara scheint im 10. Jahrhundert wieder aufgewertet worden zu sein, wie auch die Denkmäler zeigen, und steht seither an 20. Stelle in den Notitiae. 1069 ist Georgios von Patara in einem Brief Kaiser Romanos' IV. unter den Metropoliten (!) genannt ${ }^{7}$.

Überraschend und unerklärlich scheint zunächst die Stellung von Mastaura in den Notitiae episcopatuum als Protothronos der Provinz Lykia durchgehend vom 7. bis in das 12. Jahrhundert. In den Konzilsakten, die vor allem die Zeit bis in das 9. Jahrhundert betreffen, scheint Mastaura nicht auf. Die bisher noch nicht mit letzter Sicherheit lokalisierte Siedlung muß eine überragende Stellung im mittelbyzantinischen Lykien eingenommen haben. Da, wie schon im Falle von Patara, die Küstenstädte stark an Bedeutung verloren, lag Mastaura vermutlich im Landesinneren. Dort gibt es drei bedeutende landwirtschaftliche Siedlungsregionen: das Xanthos-Tal im Westen mit den Bistümern Xanthos, Tlos, Pinara und Araxa, die Kasaba Ovası im Hinterland von Myra (Quellgebiet des Myros Potamos) mit den Bistümern Arneai und Kandyba und die Elmalı Ovası (Milyas) im zentralen lykischen Hochland mit den Bistümern Podaleia, Choma und Akarassos.

Die von allen Seiten geschützte Kasaba Ovası war vermutlich aufgrund ihrer relativen Nähe zur Küste und den damit verbundenen Exportmöglichkeiten von Holz und Getreide besonders ertragreich. Durch die Schlucht des Myros Potamos führte eine nahezu ganzjährig nutzbare römische Pflasterstraße, auf der Holz (Zedern) und Getreide an die Küste transportiert werden konnten. Am Beginn der Schlucht liegt die auf altlykischen Grundmauern erbaute byzantinische Festung von Dereağż ${ }^{8}$. Nordöstlich der

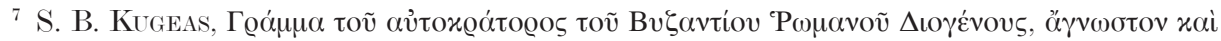

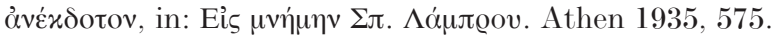

${ }^{8} \mathrm{Vgl}$. J. Morganstern (Hrsg.), The Fort at Dereağzı and other material remains in its vicinity: from antiquity to the middle ages (Istanbuler Forschungen 40). Tübingen 1993. 
Burg steht am nördlichen Flußufer die bedeutendste und größte mittelbyzantinische Basilika Lykiens ${ }^{9}$, übertroffen nur noch von der NikolaosKirche in Myra. Sowohl die Burg als auch die Kirche von Dereağzı im Zentrum der fruchtbarsten Landschaft Lykiens weisen auf eine herausragende mittelbyzantinische Siedlung, deren Name bisher nicht bekannt war. All dies spricht dafür, daß Dereağzı auch in den Notitiae episcopatuum erscheinen muß. Hier wird von Notitia 1 an (7. Jh.) durchgehend bis zur Notitia 13 (12. Jh.) an erster Stelle nach der Metropolis Myra das bisher nicht lokalisierte Bistum Mastaura angeführt, das mit Kouıбó́@oos bei

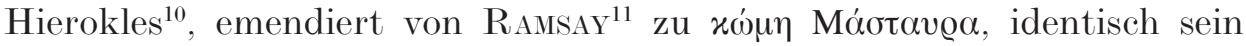

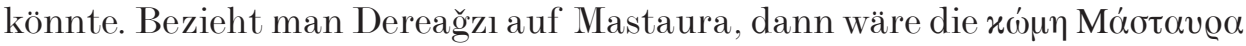
im Territorium der Metropolis Myra eigenes, ab dem 7. Jahrhundert nachweisbares, Bistum geworden und behielt nach einer umfassenden Reorganisation der Kirchenprovinz Lykia im 9. Jahrhundert den (auch durch die Denkmäler dokumentierten) ersten Rang nach Myra bis in das 12. Jahr-

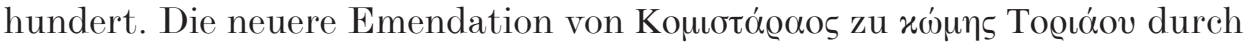
G. E. BEAN ${ }^{12}$ ist dann hinfällig; die geographisch unpassende Position der

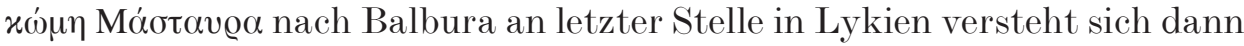

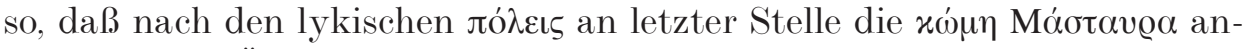
geführt ist ${ }^{13}$. Über Mastaura wurde vermutlich noch in mittelbyzantinischer Zeit Getreide aus der Kasaba Ovası nach Myra transportiert und dort im alten hadrianischen Granarium von Andriake gelagert. In der zweiten Hälfte des 10. Jahrhunderts ist Michael, epi basilikes trapezes und horrearios von Myra auf einem Siegel bezeugt ${ }^{14}$ (vielleicht horrearios des Granariums von Andriake).

An zweiter Stelle der lykischen Bistümer stand Telmessos, eine der sechs alten lykischen Metropolen. Auch Telmessos verliert als Küstenstadt seinen hohen Rang und sinkt im 10. Jahrhundert mit dem neuen Namen Makre (so benannt nach der vorgelagerten Insel Makra) auf den 15. In den Konzilsakten erscheint Telmessos 451 noch an 5. Stelle nach Myra, Makre jedoch 879 an 20.

${ }^{9}$ Vgl. J. Morganstern, The Byzantine Church at Dereağzı and Its Decoration (IstMitt Beiheft 29). Tübingen 1983.

${ }^{10}$ Le Synekdèmos d'Hiéroklès, ed. E. Honigmann. Brüssel 1939, 685, 6.

11 W. M. Ramsay, The Historical Geography of Asia Minor. London 1890, 426.

12 Anatolian Studies 10 (1960) 76.

$13 \mathrm{Vgl}$. auch die Rezension von F. Hild zu Morganstern (Hrsg.), The Fort at Dereağzı in: $J O ̈ B 46(1996) 484$.

${ }^{14}$ G. Zacos, Byzantine Lead Seals, Compiled and Edited by J. W. NesbitT, II. Bern 1984, 228; N. OIKonomidès, REB 44 (1986) 265. 
Limyra, ebenfalls eine der Metropolen, nimmt bis in das 9. Jahrhundert Rang 3 in den Notitiae ein und verschwindet dann gänzlich. An seine Stelle tritt, allerdings mit Rang 17, die benachbarte Küstenstadt Phoinix (Hafen von Limyra). In den Quellen erscheint der Hafenort Phoinix erstmals im 6. Jahrhundert n. Chr. in der Vita des Nikolaos von Sion ${ }^{15}$. Phoinix war vor allem als Ort mit einer Schiffswerft (vé́gıov) ${ }^{16}$ bekannt, wo man

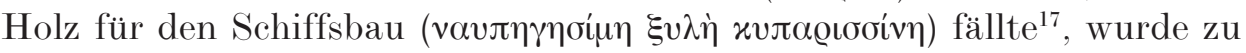
einem Flottenstützpunkt im Thema der Kibyrraioten und löste Limyra als Bistum ab. Phoinix ist als Marinebasis mit Schiffswerft der einzige Fall, in dem eine Küstenstadt neu in die Notitiae des 10. bis 12. Jahrhunderts aufgenommen wird, während fast alle anderen Küstenstädte ihren Episkopalsitz verlieren.

Araxa mit Rang 4 liegt im fruchtbaren und wasserreichen Xanthos-Tal und erreichte im 10. Jahrhundert sogar Rang 2. Es ist auch fünfmal in den Konzilsakten $(381,451,458,692,787)$ vertreten.

Aperlai (Aprilla), Zentralort einer antiken Sympolitie mit Apollonia, Isinda und Simena, liegt in einer gut geschützten, tiefen Hafenbucht. Als Bistum mit Rang 5 ist Aperlai nur bis in das 9. Jahrhundert bezeugt und verschwindet danach wie andere lykische Hafenstädte (Antiphellos, Olympos, Phaselis) aus den Notitiae. Den hohen Rang verdankt Aperlai vermutlich der wirtschaftlichen Blüte mit Purpurproduktion.

Podaleia, am Südrand der fruchtbaren Elmalı Ovası gelegen, verdankt seinen hohen 6. Rang sicher - wie Mastaura - der Landwirtschaft. In den Konzilsakten ist es 458 mit Rang 14, 536 an erster und 879 an zweiter Stelle verzeichnet. Bei der Reorganisation im 10. Jahrhundert erreichte Podaleia sogar Rang 3.

Arykanda (Orykanda) mit Rang 7 liegt wie Araxa in einer fruchtbaren Talschaft, dem Tal des Arykandos, der auch als Transportweg für den

15 Anrich, a. O. I 31; I. Šnečcenko - Nancy Patterson Š́nvčenko, The Life of Saint Nicholas of Sion. Text and Translation (The Archbishop Iakovos Library of Ecclesiastical and Historical Sources 10). Brookline, Mass. 1984, 64 (c. 37); Die Vita Nicolai Sionitae. Griechischer Text. Übersetzt und kommentiert von H. Blum. Bonn 1997, 54 (c. 37).

${ }^{16}$ Ioannis Zonarae Epitomae Historiarum libri XIII-XVIII, ed. Th. BütTner-Woвst. Bonn 1897, III 218.

17 Nikephoros, Patriarch of Constantinople, Short History. Text, Translation and Commentary by C. Mango $(C F H B 13=D O T$ 10). Washington, D.C. 1990, c. 50; Theophanis Chronographia, ed. C. DE Boor. Vol. I, Textum Graecum continens. Leipzig 1883, 385 (Übersetzung C. Mango-R. Scott, with the assistance of G. Greatrex, The Chronicle of Theophanes Confessor. Byzantine and Near Eastern History AD 284-813. Oxford 1997, 535); Georgius Cedrenus, Ioannis Scylitzae ope, ed. I. BeкKer, I-II (CSHB 13-14). Bonn 1838-1839, I 786. 
Holzeinschlag im Bergland diente. Bei der Reorganisation im 10. Jahrhundert erreichte Arykanda den Rang 4, den zuvor Araxa hatte. In den Konzilsakten erscheint Arykanda 787 und 879, hier mit dem 4. Rang. Arykanda brachte der Metropolis von Myra offensichtlich hohe Einkünfte, um die es im 14. Jahrhundert in einem Rechtsstreit ging: Die chora von Orikanta ('O@ı⿻vлí), im Tal des Bathys Potamos (Arykandos), wurde vom Patriarchen Neilos 1387 im Streit mit dem Metropoliten von Attaleia dem Metropoliten von Myra zugesprochen ${ }^{18}$.

Das bisher nicht lokalisierte Bistum Tata, Tatla (Rang 8) ist nur bis in das 9. Jahrhundert bezeugt. Möglicherweise handelt es sich um eine alte lykische Polis, die mit diesem neuen Namen Bistum wurde. Unter den bei Hierokles verzeichneten lykischen Poleis wurde nur Gagai nicht Bistum. Da eine Stadt (Gesetz Kaiser Zenons ${ }^{19}$ ) einen Bischof haben mußte, kann man vermuten, daß Gagai mit dem Namen Tata Bistum wurde ${ }^{20}$. Es gehört dann auch - wie Antiphellos, Aperlai, Olympos und Phaselis - zu den lykischen Küstenstädten, die seit dem 10. Jahrhundert nicht mehr in den Notitiae erscheinen.

Arneai, mit Rang 9 bis in das 9 . Jahrhundert bezeugt, gehörte - wie Mastaura und Kandyba - zu den landwirtschaftlichen Produktionszentren der Kasaba Ovası. Im 10. Jahrhundert verliert Arneai den Bischofssitz; zugleich rückt die Nachbargemeinde Kandyba vom 22. auf Rang 13 vor.

Die hoch oben im Kragos-Gebirge gelegene Stadt Sidyma hat bis in das 9. Jahrhundert Rang 10 und erreicht danach den 5. Rang. Sidyma war durch seine abgesonderte Lage und eine über der Stadt gelegene Festung besonders gesichert, hatte aber zugleich mit Kalabantia einen nicht weit entfernten Hafen und war auch von der Fernstraße durch das Xanthos-Tal nicht allzuweit entfernt. Der hohe Rang wird auch in den Konzilsakten deutlich. 359 erscheint Pinara mit Sidyma vereint an erster Stelle nach Patara, 458 hat Sidyma schon Rang 9 gegenüber von Pinara mit Rang 20, 681 steht Sidyma an erster Stelle nach Myra und erscheint 692 an der Spitze der lykischen Bistümer, 787 hingegen nur mehr an sechster Stelle.

Anstelle des im 10. Jahrhundert auf Rang 5 gehobenen Bistums Sidyma erscheint nun das noch nicht lokalisierte Bistum Hagiodula an 10. Stelle in den Notitiae.

${ }_{18}$ MM II 92-95, Nr. 388, 389; J. Darrouzès, Les Regestes des Actes du Patriarcat de Constantinople. Vol. I. Les Actes des Patriarches, Fasc. VI. Paris 1979, Nr. 2812, 2813; Barbara Flemming, Landschaftsgeschichte von Pamphylien, Pisidien und Lykien im Spätmittelalter (Abh. für die Kunde des Morgenlandes 35,1). Wiesbaden 1964, 108.

${ }^{19}$ Cod. Iust. I 3, 35 (36); vgl. unten Zenonupolis.

${ }^{20}$ Von Ruge, Gagai. RE 7/1 (1910) 465f. wird Tata mit Gagai identifiziert. 
An 11. Stelle steht Zenonupolis. Da in der dicht besiedelten lykischen Landschaft eine dynastische Neugründung kaum möglich ist, wurde vermutlich eine ältere Siedlung umbenannt, vielleicht Apollonia. Die bedeutende antike Siedlung (mit Theater!) hätte sich dann aus der Sympolitie mit Aperlai gelöst und wurde als selbständige Polis auch Bistum (Gesetz Zenons ${ }^{21}$ ). Eine Kreuzkuppelkirche bezeugt das Fortleben im Mittelalter.

Olympos, an 12. Stelle, gehörte wie Patara, Pinara, Tlos, Xanthos und Myra zu den sechs Städten im Lykischen Bund (1. Jh. v. Chr.), die drei Stimmen hatten ${ }^{22}$. In den Seeräuberkriegen 77 v. Chr. von P. Servilius Vatia (Isauricus) erobert und zerstört, verlor es seinen hohen Rang und gehört nicht mehr zu den sechs kaiserzeitlichen Metropolen Lykiens (Myra, Limyra, Patara, Tlos, Telmessos, Xanthos). Die alte Bergstadt wurde vom Berg Olympos an die Küste verlegt. An der Stelle der alten Stadt Korykos entstand die neue Stadt Olympos, die auch Bistum wurde. Als Bischofssitz des Kirchenvaters Methodios hat Olympos zwar Berühmtheit erlangt, den hohen alten Rang hat die Stadt aber nicht mehr erreicht. Mehrfach in den frühen Konzilsakten bezeugt $(431,458,518)$ begegnet Olympos nicht mehr in den späteren Akten und scheidet im 10. Jahrhundert wie die anderen lykischen Hafenstädte Phaselis, Aperlai und Antiphellos als Bistum aus den Notitiae aus.

Mit Rang 13 ist die alte lykische Metropolis Tlos ebenfalls weiter hinten gereiht als zu erwarten. In den Konzilsakten ist Tlos häufig vertreten: 451, 458, 536, 787 und 879 (hier nur mehr mit Rang 16). Nach dem Ausscheiden von Olympos führt Tlos Rang 12.

Korydalla mit Rang 14 blieb trotz der großen Nähe zur Küste als Zentralort in der außerordentlich fruchtbaren Schwemmlandebene des Alakır Çayı Bistum, nach der Reorganisation im 10. Jahrhundert mit Rang 24. In den Konzilsakten ist Korydalla wie Tlos häufig verzeichnet: 451, 458, 787 (hier an erster Stelle nach Myra) und 879 mit Rang 3.

Kaunos mit Rang 15 ist ein Sonderfall. Die ursprünglich karische Stadt, eine der bedeutendsten an der kleinasiatischen Westküste, wurde erst bei der diokletianisch-konstantinischen Reichsreform in die neue Provinz Lykia eingegliedert. Bis in das 9. Jahrhundert mußte sich das neu in die lykischen Poleis integrierte Bistum mit Rang 15 begnügen, stieg aber bei der Reorganisation im 10. Jahrhundert mit dem Beinamen Hagia auf Rang 6 auf und überholte dabei alle alten lykischen Metropolen außer der Metropolis Myra. Auf Rang 15 sinkt dagegen die alte lykische Metropolis Telmessos mit dem neuen Namen Makre.

${ }^{21}$ Cod. Iust. I 3, 35 (36); vgl. oben Gagai/Tata.

${ }^{22}$ Strabon XIV 3. 
Akarassos mit Rang 16 ist ein bisher nicht lokalisiertes Bistum, kann aber nun gemäß dem Stadiasmos von Patara, einem inschriftlich erhaltenen Straßenverzeichnis aus der Zeit des Kaisers Claudius, mit Elmalı im zentralen lykischen Hochland identifiziert werden. Elmalı (Almaly), im Zentrum der landwirtschaftlich außerordentlich produktiven Ebene von Elmalı gelegen, wurde in osmanischer Zeit zum bevölkerungsreichsten Siedlungs- u. Marktort in Lykien. Akarassos, schon in den Konzilsakten von 451, 458 und 879 vertreten, erfuhr bei der Reorganisation im 10. Jahrhundert eine Aufwertung auf Rang 9. Auf Rang 16 erscheint seit dem 10. Jahrhundert das Bistum Phileta, das bis dahin nur durch Bischof Germanos bekannt war, der 879 am Konzil von Konstantinopel teilnahm. Eine Grenzinschrift lokalisiert nun Phileta im Norden von Elmalı.

Xanthos mit Rang 17 ist auch in den Konzilsakten von 381, 458 und 692 vertreten. Im 10. Jahrhundert auf Rang 7 erhoben, ist Xanthos die einzige der alten lykischen Metropolen - außer Myra -, die in den Notitiae einen hohen Rang erreichte. Die Stadt hat zwar im 7. Jahrhundert durch den Zusammenbruch des Seehandels an Bedeutung verloren, wurde dann aber durch ihre günstige Lage am Ausgang des fruchtbaren Xanthos-Tals und Anfang des Xanthos-Deltas zum zentralen Siedlungsort. Diese Rolle hat auch der heutige Nachfolgeort Kınık übernommen, so benannt nach einem Turkmenenstamm, der sehr früh (schon 12. Jh.) hier siedelte. Den Rang 17 von Xanthos übernahm im 10. Jahrhundert Phoinix, das Limyra mit Rang 3 ablöste.

Die im nordwestlichen lykischen Hochland gelegene Stadt Bubon/ Bobos, auch Sophianupolis, behält, seit dem 10. Jahrhundert mit dem neuen Namen Proine, durchgehend Rang 18. Bubon ist auch in den Akten von 381,451 und 458 vertreten.

Die westlich von Kaunos in einer tiefen, gut geschützten Hafenbucht (Karaağaç Körfezi) gelegene Stadt Markiane mit Rang 19 erhält im 10. Jahrhundert Rang 11. Auf Rang 19 rückt Balbura von Rang 34/35 vor. In den Akten von 518 ist Markiane an erster Stelle vor Eudokias, Olympos und Korydalla genannt. Markiane, wie Eudokias eine dynastische Gründung des 5. Jahrhunderts, hatte damals noch einen besonders hohen Rang. Seine eigentliche Bedeutung verdankte Markiane der sicheren Lage, hatte jedoch kaum landwirtschaftlich nutzbares Hinterland. Die Kaufleute aus Bari, die 1087 die Reliquien des H. Nikolaos von Myra nach Bari brachten, legten

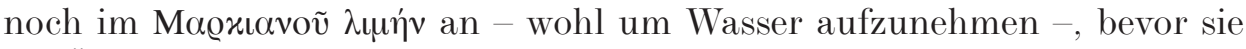
die Ägäis durchquerten ${ }^{23}$.

${ }^{23}$ Anrich, a. O. I 442f. 
Oinoanda (Oinunda), wie Bubon und Balbura im nordwestlichen lykischen Hochland gelegen, hatte mit dem Epikureer Diogenes, dessen philosophisches Werk inschriftlich in Oinoanda erhalten ist, eine glänzende kulturelle Vergangenheit. In den Notitiae wird es im 10. Jahrhundert von Rang 20 auf Rang 14 aufgewertet. Rang 20 erhält Patara.

Rang 21 hält bis in das 9. Jahrhundert Choma, danach Rang 22. An die 21. Stelle rückt von Rang 32/33 Komba. Choma gehört mit Podaleia und Akarassos zu den landwirtschaftlich bedeutenden Zentren der Ebene von Elmalı, an deren Südwestrand Komba lag. Es ist in den Akten von 381, 431, 451 und 458 an auffälliger erster Stelle nach Myra genannt, in den Akten von 879 jedoch nur mehr an 12. Stelle nach Myra (unter 22 lykischen Konzilsteilnehmern).

Das hoch über seinem Hafen Antiphellos gelegene Phellos führt in Notitia 1 Rang 22, danach Rang 23. An seine Stelle tritt in Notitia 2 Kandyba. Der vor allem in der Forstwirtschaft (Zedern) bedeutende Ort rückte im 10. Jahrhundert auf Rang 13 vor und ist auch in den Akten von 458 (an letzter Stelle), 787 (mit Rang 8) und 879 (mit Rang 10) vertreten.

Antiphellos, bedeutend in der Antike durch Schwammfischerei, mit Rang 23/24 verschwindet als Hafenstadt wie Aperlai, Phaselis und Olympos im 10. Jahrhundert aus den Notitiae. Es erscheint auch in den Akten von 451 und 458. Auf Rang 24 sinkt im 10. Jahrhundert Korydalla von Rang 14.

Rang 24/25 nimmt bis in das 9 . Jahrhundert die bedeutende Hafenstadt Phaselis (einzige literarisch bezeugte griechische Kolonie Lykiens) ein, die wie Antiphellos, Aperlai und Olympos im 10. Jahrhundert aus den Notitiae verschwindet. Phaselis ist auch in den Akten von 381, 451, 458 und 787 bezeugt. An seine Stelle tritt mit Rang 25 im 10. Jahrhundert das Bistum Lornaia in Nordwestlykien; Lornaia ist erstmals in den Akten von 879 bezeugt (an 5 . Stelle nach Myra bei 22 lykischen Konzilsteilnehmern).

Rodiapolis auf Rang 25/26 ist ebenfalls nur bis in das 9. Jahrhundert Bistum. Es erscheint auch in den Akten von 518. An seine Stelle tritt im 10. Jahrhundert Pinara mit Rang 26. Der niedrige Rang der bedeutenden alten lykischen Stadt (mit drei Stimmen im Lykischen Bund) ist schwer zu verstehen, ebenso, warum Pinara erst im 10. Jahrhundert in die Notitiae Eingang fand, obwohl es schon 359 (damals mit Sidyma vereint), 458, 692, 787 und 879 in den Akten erscheint (auch hier schon jeweils mit sehr niedrigem Rang). Möglicherweise war das Verhältnis des Klerus von Pinara mit dem übrigen Provinzialklerus von Lykien gespannt. Hosios Nikolaos von Hagia Sion baute als Bischof von Pinara um 550 gegen den 
Widerstand der Stadtbeamten und des örtlichen Klerus eine TheotokosKirche $^{24}$.

Rang 26/27 nimmt die entlegene Gebirgsstadt Akalissos im südlichen Lykien ein, einst Vorort einer Sympolitie mit Idebessos und Korma. In den Akten ist Akalissos 458 bezeugt. Im 10. Jahrhundert tritt an seine Stelle mit Rang 27 das noch nicht lokalisierte Bistum Tergasos (schon 879 in den Akten bezeugt).

Lebissos mit Rang 27/28 sank im 10. Jahrhundert auf Rang 33. Der niedrige Rang des bedeutenden Bistums, das alle anderen lykischen Suffraganbistümer bis weit in die osmanische Zeit überlebte, erklärt sich aus seiner Abhängigkeit von Telmessos/Makre, in dessen Chora es selbständiges Bistum wurde. Als griechische Enklave mit einheimisch kleinasiatischem Dialekt ${ }^{25}$ blieb Lebissos (heute Kayaköy) bis 1922 bestehen. Rang 28 erhält im 10. Jahrhundert Eudokias (zuvor 30/31).

Rang 28/29 hält bis in das 9. Jahrhundert das ebenfalls noch nicht lokalisierte Bistum Akanda; im 10. Jahrhundert sinkt es auf Rang 32. Akanda ist auch in den Akten von 458 erwähnt. Rang 29 erhält im 10. Jahrhundert Nysa, das zuvor Rang 33/34 hatte. Nysa ist auch in den Akten von 787 genannt.

Rang 29/30 wird von Palaioton (vielleicht neuer Name von Gagai) durchgehend bis in das 12. Jahrhundert eingenommen.

Eudokias, eine dynastische Neugründung des 5. Jahrhunderts, ist bei Hieroklēs zwischen Kandyba und Patara verzeichnet ${ }^{26}$, lag also vermutlich in der Gegend von Kalamin (Kalkan) und ist vielleicht mit Tymena (Köybaşı) an der Straße von Kalkan nach Elmalı zu identifizieren. Die Stadt hält Rang 30/31 und steigt im 10. Jahrhundert auf Rang 28 auf. Zugleich wird das Bistum Meloeton (vielleicht Melanippe) von Rang 36 auf Rang 31 erhoben.

Patara mit Rang 31/32 steigt im 10. Jahrhundert auf Rang 20.

Komba mit Rang 32/33 steigt im 10. Jahrhundert auf Rang 21.

Nysa mit Rang 33/34 steigt im 10. Jahrhundert auf Rang 29.

Balbura mit Rang 34/35 steigt im 10. Jahrhundert auf Rang 19. Balbura ist auch in den Akten von 451, 458 und 879 vertreten.

Meloeton mit Rang 35/36 steigt im 10. Jahrhundert auf Rang 31.

Kyaneai mit Rang 36/37 erreicht im 10. Jahrhundert den hohen Rang 8. Auch in den Akten von 879 ist Kyaneai nicht mehr an letzter Stelle ge-

${ }^{24}$ Anrich, a. O. 50; I. Ševčenko -Nancy Patterson Ševč́nko, a. O. 102 (c. 69); Blum, a. O. $82-84$ (c. 69$)$.

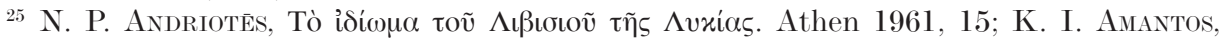
Laographia 7 (1923) 336f.

${ }^{26}$ Honigmann, a. O. 684, 9 . 
nannt, sondern hält Rang 11 unter 22 Suffraganbistümern. Im 14. Jahrhundert gehört Kyaneai gemeinsam mit Makre/Lebissos zu den lykischen Suffraganbistümern, die noch im Patriarchatsregister aufscheinen. Ein groß angelegter, flächendeckender Survey der Chora von Kyaneai (Leitung F. Kolb, Tübingen) zeigt anhand vieler mittelbyzantinischer Kirchenbauten, daß die heute nahezu unbevölkerte Region vermutlich bis in das 14. Jahrhundert dicht besiedelt und bebaut war.

Eine Analyse der Rangordnung der lykischen Bistümer läßt Rückschlüsse auf die Siedlungsgeschichte in byzantinischer Zeit zu.

Von den sechs alten lykischen Metropolen (Myra, Limyra, Patara, Xanthos, Tlos und Telmessos) und ebenfalls sechs Städten mit drei Stimmen im lykischen Bund (Pinara, Tlos, Xanthos, Patara, Myra und Olympos) erscheinen sieben von acht in der Notitia 1: Myra (Metropolis), Telmessos mit Rang 2, Limyra Rang 3, Olympos Rang 12, Tlos Rang 13, Xanthos Rang 17 und Patara Rang 31. In der Notitia 7 des 10. Jahrhunderts erscheinen nur mehr sechs von acht: Myra, Xanthos mit Rang 7, Tlos mit Rang 12, Telmessos mit dem neuen Namen Makre Rang 15, Patara mit Rang 20 und Pinara mit Rang 26. Auffällig ist besonders das Ausscheiden der Hafenstadt Olympos und der wesentlich niedrigere Rang von Telmessos/ Makre. Der Zusammenbruch des Seehandels im 7. Jahrhundert zeigt sich hier deutlich. Auch andere wichtige Hafenstädte, so Phaselis, Aperlai und Antiphellos verschwinden aus den Notitiae. Das Bistum von Limyra wandert dagegen zur nahen Hafenstadt Phoinix, die mit ihrer Schiffswerft eine Flottenbasis des Themas der Kibyrraioten wurde.

Lykien muß sich seither auf Land- und Forstwirtschaft beschränken. Den Holzexport bezeugt ausdrücklich Marino Sanudo um 1300 für Kau$\operatorname{nos}^{27}$. Der Fernverkehr von Myra nach Zypern und in die Levante wurde eingestellt, wie wir aus der Vita des Konstantinos Iudaios wissen; dieser pilgert zur Zeit Basileios' I. (867-886) auf dem Landweg von Bithynien zum Grab des H. Nikolaos in Myra. Für seine Weiterreise nach Zypern ging er - da er offensichtlich in Myra keine Schiffspassage finden konnte - auf dem Landweg nach Attaleia; von dort nahm man zu dieser Zeit das Schiff nach Zypern ${ }^{28}$.

${ }^{27}$ K. Kretschmer, Die italienischen Portolane des Mittelalters (Veröff. Inst. f. Meereskunde u. Geogr. Inst. Univ. Berlin 13). Berlin 1909, 246: Prepia (gemeint ist Kaunos) habet aestivo tempore bonum portum, dum ex parte terrae securitas habeatur. Deinde intratur flumen; quod septem pedum altitudinis habet aquam. Hocque modo onerantur navigia, quae deferunt lignamina in Aegyptum.

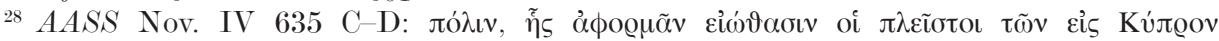

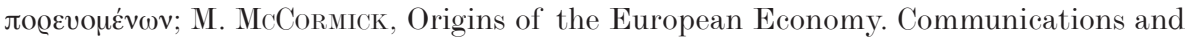
Commerce, A.D. 300-900. Cambridge 2001, 424, 426. 
Bistümer in land- und forstwirtschaftlich ergiebigen Regionen erhielten in den Notitiae von Anfang an einen höheren Rang als die alten lykischen Metropolen. Zuvor nur eine Kome in der Chora von Myra erwirbt Mastaura durch seine Schlüsselposition in der Kasaba Ovası den ersten Rang unter den lykischen Bistümern. Auch die weiteren Bistümer der Kasaba Ovası weisen hohe Ränge auf: Arneai Rang 9 und Kandyba Rang 13. Das abgelegene, vorher kaum bekannte Araxa, im oberen Xanthos-Tal erhält Rang 4, im 10. Jahrhundert sogar Rang 2.

Die im zentralen lykischen Hochland gelegene Elmalı Ovası gewinnt ebenfalls an Bedeutung ${ }^{29}$. Die alte hellenistische Stadt Podaleia im Süden der Ebene erhält Rang 6, seit dem 10. Jahrhundert Rang 3. Die ebenfalls schon hellenistische Stadt Choma erhält Rang 21/22, Komba wird im 10. Jahrhundert von Rang 32/33 auf Rang 21 und die zuvor nur aus dem Stadiasmos von Patara und der Vita des Hosios Nikolaos Sionites bekannte Stadt Akarassos/ Elmalı von Rang 16 auf Rang 9 aufgewertet.

Die Bergstadt Arykanda im oberen Arykandos-Tal war ein Zentrum der Forstwirtschaft. Von hier wurde das Holz für den Schiffsbau zur Werft nach Phoinix geflößt. Ursprünglich auf Rang 7 erhält Arykanda im 10. Jahrhundert Rang 4.

Die alten lykischen Metropolen des Xanthos-Tales: Xanthos und Tlos sowie Pinara (Stadt mit drei Stimmen) erscheinen in den Notitiae nicht mehr unter den ersten 10 Bistümern. Nur Xanthos erreicht im 10. Jahrhundert wieder einen hohen Rang (7). Pinara könnte wegen seiner feindlichen Haltung gegenüber Hosios Nikolaos Sionites seinen hohen Rang verloren haben, und Tlos hatte 542 einen Rechtsstreit mit dem Bischof von Telmessos. Xanthos hingegen erhielt wieder seinen hohen Rang.

Der eingangs geforderten Überprüfung der Notitiae durch die Geschichte ihrer Entstehungszeit und durch die Konzilsakten halten die lykischen Notitiae nicht nur durchaus stand, sondern werden selbst zu einem wichtigen Zeugnis der politischen Entwicklung. Der Wandel vom seeorientierten Land mit seiner zentralen Rolle in der Schiffahrt des östlichen Mittelmeerraumes $^{30}$ zum Binnenland, das allerdings noch Holz und Getreide exportierte, wird schon in den frühen Notitiae des 7. Jahrhunderts spürbar.

${ }^{29}$ Zur Elmalı Ovası (Milyas) vgl. zuletzt M. AdAK - S. ŞAHin, Das römische Strassen- und Siedlungssystem in der lykischen Milyas (Materialien zur Bodendenkmalpflege im Rheinland 16). Bonn 2004.

${ }^{30}$ Paulus stieg 57 n. Chr. in Patara von einem Schiff aus Philippi auf ein Schiff nach Phoenikien um: Act. Ap. 21, 1-2. Auf seiner Fahrt von Caesarea in Palästina fuhr der Apostel 59 n. Chr. auf einem Schiff aus Adramyttion über Sidon nach Myra; dort wechselte er auf einen alexandrinischen Getreidefrachter: Act. Ap. 27, 5-6. 
Eklatant sichtbar wird der Wandel aber erst in der Notitia 7 des frühen 10. Jahrhunderts. Fast alle alten Hafenstädte verschwinden aus den Notitiae. Phoinix wird als Marinebasis mit Schiffswerft neues Bistum und Telmessos fällt mit dem neuen Namen Makre von Rang 2 auf Rang 15 zurück. Telmessos/Makre war wohl ebenfalls ein Stützpunkt der Kibyrraioten, der wahrscheinlich unter Kaiser Anastasios II. (713-715) eingerichtet wurde (Telmessos trägt in Notitia 2 den Beinamen Anastasiupolis). Eine Ausnahme ist Kaunos, das mit dem neuen Namen Hagia von Rang 15 auf Rang 6 vorrückt (Holzexporthafen).

Der in der Notitia 7 bezeugte radikale Bruch fand in der Tat erst um die Wende vom 9. zum 10. Jahrhundert statt, wie uns die Konzilsakten von 879 bestätigen. Hier sind zwar schon die neuen Bistümer Lornaia, Tergasos, Phileta, Makre (als Nachfolger von Telmessos) und Hagiodula vertreten, daneben hält aber Limyra den 1. Rang nach Myra. Da Limyra in der Notitia 7 nicht mehr vertreten ist, erfolgte die Fixierung der Neuordnung zwischen 879 und 901/907, der Entstehungszeit von Notitia 7.

Eine Sonderstellung nimmt unter den Notitiae die Notitia 3 ein, die auch auf antike Städtelisten zurückgreift ${ }^{31}$. In Lykien wird allerdings nur die Notitia 2 kopiert und an letzter Stelle Tragalasos hinzugefügt. Tragalassos war eine hellenistische Polis im Hinterland von Myra, wurde jedoch in der Kaiserzeit eine Kome in der Chora von Myra. Als solche erscheint sie in der Vita des Hosios Nikolaos von H. Sion. Eine Verwechslung mit Sagalassos, wie in der Ausgabe von Darrouzès vermutet, ist sicher auszuschließen.

${ }^{31}$ So auf die Städteliste des Hierokles, ed. Honigmann, a. O.; vgl. Eleonora Kuntura-

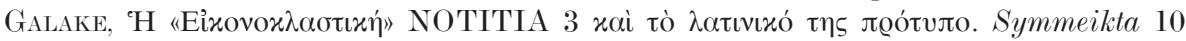
(1996) $47 \mathrm{f}$. 


\section{Lykien in den Notitiae ePiscopatudm}

\begin{tabular}{|c|c|c|c|c|c|c|c|}
\hline $\begin{array}{l}\text { Not. } 1 \\
7 . \text { Jh. } \\
\text { (Herakleios) }\end{array}$ & $\begin{array}{l}\text { Not. } 2 \\
\text { spätestens } \\
\text { 1. Viertel } \\
\text { 9. Jh. }\end{array}$ & $\begin{array}{l}\text { Not. } 3 \\
\text { 2. Hälfte - } \\
\text { Ende 9. Jh. }\end{array}$ & \begin{tabular}{|l} 
Not. 4 \\
nahe dem \\
Konzil 869
\end{tabular} & $\begin{array}{l}\text { Not. } 7 \\
\text { zwischen } \\
901 \text { und } \\
907\end{array}$ & $\begin{array}{l}\text { Not. } 9 \\
\text { Archetyp } \\
\text { im 10. Jh. }\end{array}$ & $\begin{array}{l}\text { Not. } 10 \\
\text { vor } 997 \\
\text { oder vor } \\
1020 / 1030\end{array}$ & $\begin{array}{l}\text { Not. } 13 \\
12 . \mathrm{Jh} .\end{array}$ \\
\hline Myra & Myra & Myra & Myra & Myra & Myra & Myra & Myra \\
\hline 1 Mastaura & Mastaura & Mastaura & Mastaura & Mastaura & Mastaura & Mastaura & Mastaura \\
\hline 2 Telmēsos & $\begin{array}{l}\text { Telmissos } \\
\text { Anastasiu- } \\
\text { polis }\end{array}$ & Telmissos & Telmēssos & Araxa & Araxa & Araxa & Araxa \\
\hline 3 Limyra & Limyra & Limyra & Limyra & Podalia & Podalia & Podaleia & Podaleia \\
\hline 4 Araxa & Araxa & Araxa & Araxa & Orykanda & Orykandos & Orykandos & Orykanda \\
\hline 5 Aprilla & Aprilla & Aprila & Aprilla & Sidyma & Sidyma & Sidyma & Sidyma \\
\hline 6 Podaleia & Podalia & Podaleia & Podaleia & \begin{tabular}{|l} 
Kaunos \\
Hagias
\end{tabular} & $\begin{array}{l}\text { Skamnōn } \\
\text { Hagias }\end{array}$ & $\begin{array}{l}\text { Skamnōn } \\
\text { Hagias }\end{array}$ & \begin{tabular}{|l} 
Kaunos \\
Hagias
\end{tabular} \\
\hline 7 Orykanda & Arykanda & Arykanda & Orykanda & Xanthos & Xanthos & Xanthos & Xanthos \\
\hline 8 Tata & Tata & Tata & Tatla & Kainea & Kenea & Kenea & Kainea \\
\hline 9 Arnea & Arnea & Arnea & Arnea & Akarassos & Agarassos & Aigarassos & Akrassos \\
\hline 10 Sidyma & Sidyma & Sidyma & Sidyma & Hagiodula & Hagiodula & Hagiodula & Hagiodula \\
\hline $\begin{array}{l}11 \text { Zēnōnu- } \\
\text { polis }\end{array}$ & $\begin{array}{l}\text { Zēnōnos } \\
\text { poleōs }\end{array}$ & Zēnōnupolis & Zēnōnupolis & Markianē & Markianē & Markianē & Markianē \\
\hline 12 Olympos & Olympos & Olympos & Olym & Tlō & Tlōn & Tlōn & Tlōn \\
\hline 13 Tlōn & Tlōn & Tlōn & Tlōn & Kandiba & Kandiba & Kandiba & Kandyba \\
\hline $\begin{array}{l}14 \text { Kory- } \\
\text { dala }\end{array}$ & Korydalla & Korydala & Korydalla & Oinianda & Oinianda & Oinianda & $\begin{array}{l}\text { Oinunda } \\
\text { Mokondōn }\end{array}$ \\
\hline 15 Kaunos & Kaunos & Kaunos & \begin{tabular}{|l} 
Kaunos \\
Aleias
\end{tabular} & Makrē & Makrē & Makrē & Makrē \\
\hline $\begin{array}{l}16 \text { Akaras- } \\
\text { sos }\end{array}$ & Akrassos & Akrassos & Akrassos & Philēta & Philēta & Philēta & Philēta \\
\hline 17 Xanthos & Xanthos & Xanthos & Xanthos & Phoinix & Phoinikōn & Phoinikōn & Phoinix \\
\hline $\begin{array}{l}18 \text { Bobos } \\
\text { Sophianu- } \\
\text { polis }\end{array}$ & $\begin{array}{l}\text { Bobos } \\
\text { Sophianu- } \\
\text { polis }\end{array}$ & $\begin{array}{l}\text { Bobos } \\
\text { Sophianu- } \\
\text { polis }\end{array}$ & $\begin{array}{l}\text { Bobos } \\
\text { Sophianu- } \\
\text { polis } \\
\text { (Prōana) } \\
\end{array}$ & Prōinē & Prōinē & Prōinē & Prōinē \\
\hline \begin{tabular}{|l|}
$\mathbf{1 9}$ \\
Markianē
\end{tabular} & Markianē & Markianē & Markianē & Balbura & Barbylōn & Barbylōn & Barbylōn \\
\hline 20 Oinunda & Oinunda & Oinunda & Oinunda & Patara & Patara & Patara & Patara \\
\hline 21 Chōma & Chōma & Chōma & Chōma & Komba & Komba & Komba & Komba \\
\hline 22 Phellos & Kandyba & Kandyba & Kandyba & Chōma & Chōma & Chōma & Chōma \\
\hline \begin{tabular}{|l}
23 Anti- \\
phellos
\end{tabular} & Phellos & Phellos & Phellos & Phellos & Phellos & Phellos & Phellos \\
\hline 24 Phasēlis & Antiphellos & Antiphellos & Antiphellos & Korydalla & Korydalla & Korydala & Korydalla \\
\hline $\begin{array}{l}25 \text { Rodia- } \\
\text { polis }\end{array}$ & Phasēlis & Phasēlis & Phasēlis & Lornaia & Lornea & Lornea & Lornaia \\
\hline 26 Akalissos & Rodiapolis & Rodiapolis & Rodiapolis & Pinara & Pinara & Pinara & Pinara \\
\hline \begin{tabular}{|l|}
27 Lebissos \\
\end{tabular} & Akalissos & Akalissos & Akalissos & Tergasos & Tergasos & Tergasos & Tergasos \\
\hline
\end{tabular}




\begin{tabular}{|c|c|c|c|c|c|c|c|}
\hline \begin{tabular}{|l|} 
Not. 1 \\
7. Jh. \\
(Herakleios)
\end{tabular} & $\begin{array}{l}\text { Not. } 2 \\
\text { spätestens } \\
\text { 1. Viertel } \\
9 . \text { Jh. } \\
\end{array}$ & $\begin{array}{l}\text { Not. } 3 \\
\text { 2. Hälfte - } \\
\text { Ende 9. Jh. }\end{array}$ & \begin{tabular}{|l} 
Not. 4 \\
nahe dem \\
Konzil 869
\end{tabular} & $\begin{array}{l}\text { Not. } 7 \\
\text { zwischen } \\
901 \text { und } \\
907\end{array}$ & $\begin{array}{l}\text { Not. } 9 \\
\text { Archetyp } \\
\text { im 10. Jh. }\end{array}$ & $\begin{array}{l}\text { Not. } 10 \\
\text { vor } 997 \\
\text { oder vor } \\
1020 / 1030\end{array}$ & $\begin{array}{l}\text { Not. } 13 \\
12 . \mathrm{Jh} .\end{array}$ \\
\hline 28 Akanda & Lebissos & Lebissos & Lebissos & Eudokias & Eudokias & Eudokias & Eudokias \\
\hline $\begin{array}{l}29 \text { Palaiō- } \\
\text { tōn }\end{array}$ & Akanda & Akanda & Akanda & Nysa & Nēsos & Nēsos & Nysa \\
\hline $\begin{array}{l}30 \text { Eudo- } \\
\text { kias Iusti- } \\
\text { nianupolis } \\
\end{array}$ & $\begin{array}{l}\text { Palaiōtōn } \\
\text { Iustinianu- } \\
\text { polis }\end{array}$ & Palaiōtōn & Palaiōtōn & Palaiōtōn & Palaiōtōn & Paliōtōn & Palaiōtōn \\
\hline 31 Patara & Eudokias & Eudokias & Eudokias & Mēloētōn & Mēlōtōn & Mēlōtōn & Mēloètōn \\
\hline 32 Komba & Patara & Patara & Patara & Akanda & Akanda & Akanda & Akanda \\
\hline 33 Nysa & Komba & Komba & Komba & Lebissos & Lebissos & Lebissos & Lebissos \\
\hline 34 Balbura & Nysa & Nysa & Nysa & & & & \\
\hline 35 Mēloētōn & Balbura & Balbura & Balbura & & & & \\
\hline 36 Kyanea & Mēlö̈tōn & Mēloētōn & Mēloētōn & & & & \\
\hline \multirow[t]{2}{*}{37} & Kyanea & Kyanea & Kyanea & & & & \\
\hline & & [Tragalasos] & & & & & \\
\hline
\end{tabular}




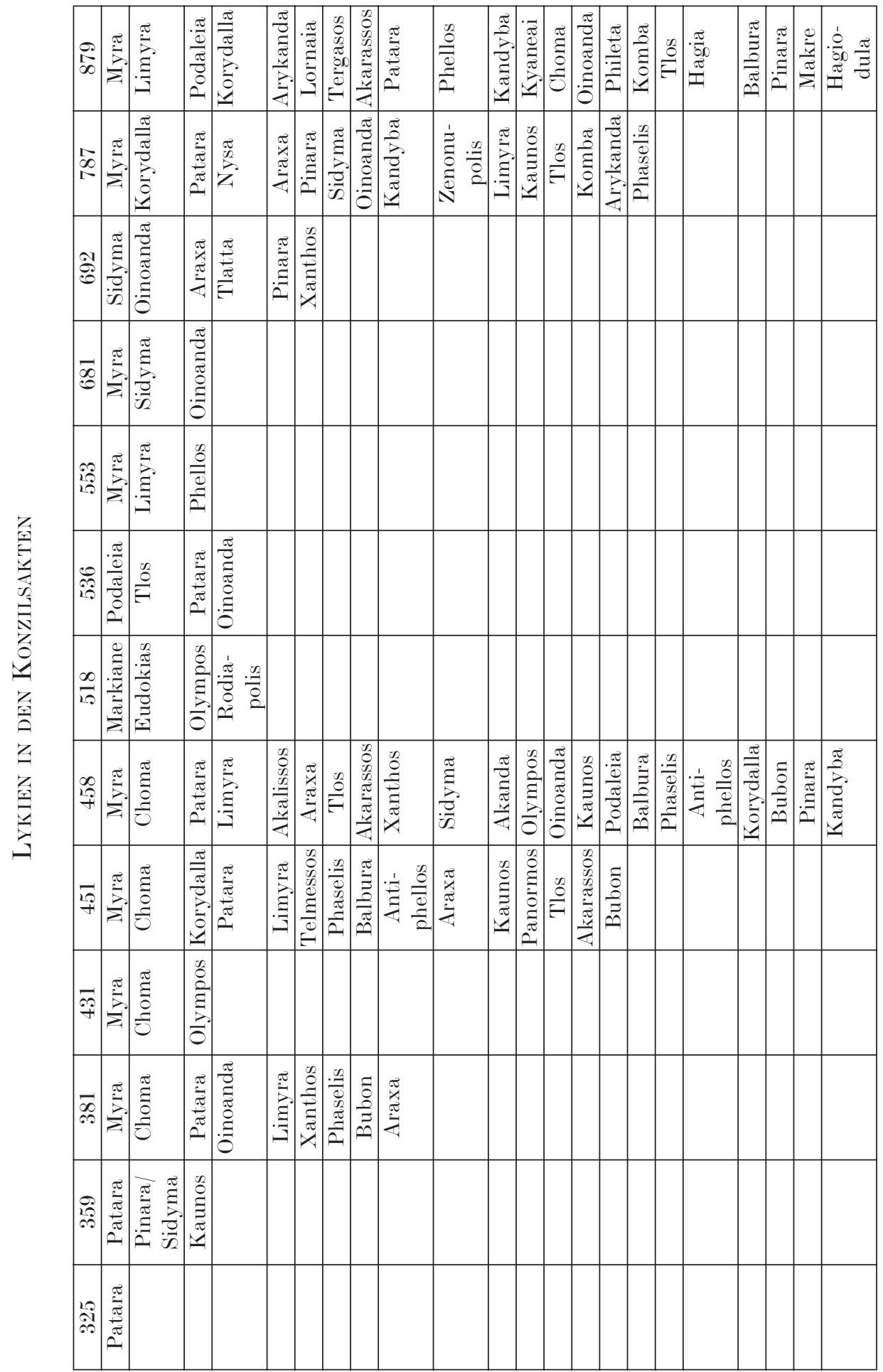




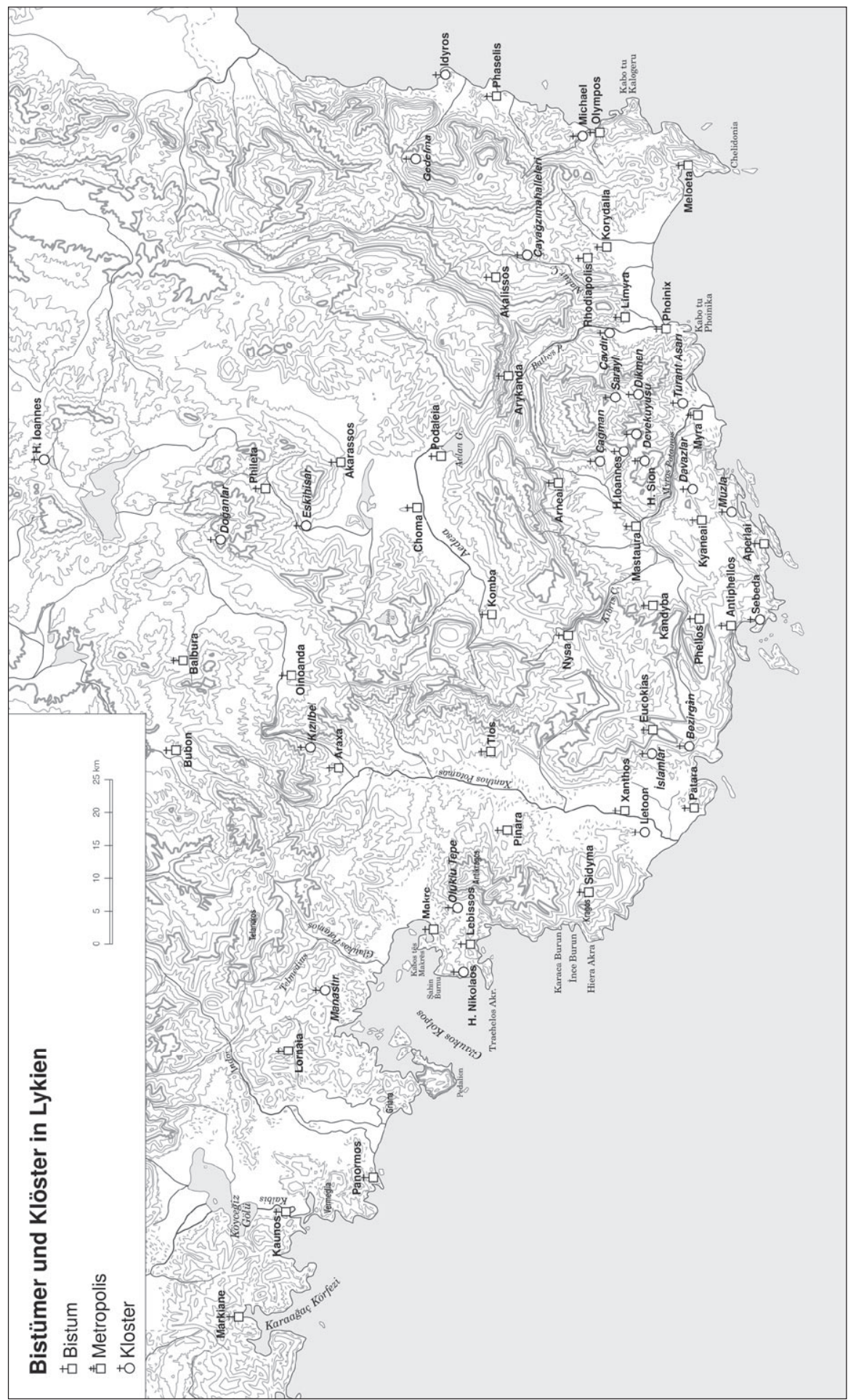


